\title{
A STUDY OF THE DISTRIBUTION \\ OF INTERSTELLAR MATTER
}

\author{
LARS OLOF LODÉN \\ Astronomical Observatory, Uppsala, Sweden
}

\begin{abstract}
As a first step towards an empirical estimation of the minimum size of interstellar dust formation units (clouds etc.) the correlation between angular separation and difference in colour excess has been studied for a selection of close star couples with coinciding magnitude and spectral type. Principally, the result indicates that there is no palpable correlation between the difference in colour excess and the lateral separation of the stars. This and other properties speak in favour of the conception that obscuring matter in the Milky Way is concentrated in extremely small units of high density.
\end{abstract}

\section{Introduction}

The convenient picture of the interstellar medium as being more or less homogeneously distributed over considerably extended parts of the Milky Way is in any respect unallowably oversimplified. Several astrophysical phenomena would be explained in a relatively natural way if the interstellar matter was considered to be concentrated in numerous very small and dense elementary units in the shape of miniclouds, bars, or rods. The most conspicuous indication is apparently the capricious behaviour of the colour excess. It is a well-known fact that in no part of the sky can there be found a good point-to-point correlation between distance and colour excess for the stars. Only a very vague statistical correlation may be established, which will differ appreciably between various parts of the sky. For numerous more or less nearby stars there has been recorded an astonishingly great excess, while for some very distant ones the excess is practically undetectable. This phenomenon is also reflected by the correspondingly great variability in the specific colour excess (units of magnitude per kpars). Firstly there is a general tendency for this parameter to vary strongly with distance in a certain direction, independently of its absolute level for a given distance. In some cases the observed specific excess will diminish with a power of ten when the distance increases from $0.4 \mathrm{kpc}$ to $4 \mathrm{kpc}\left(m_{0}-M=8\right.$ to 13), thus indicating that most of the extinction might occur within a relatively short distance from the observer. In general, the variation is more modest. Table I illustrates two different instances. Secondly there is a tremendous amplitude in the individual values of the specific excess. A reasonable average value for $1 \mathrm{kpars}$ in the galactic belt will lie around 0.25 , but the individual values range between 0.03 and 5.5 or even more. At least a few per cent of the early-type stars beyond 1 kpars near the galactic plane show values around 0.05 and the same fraction of the more local ones show values greater than 2.5. Certain checks show that these discrepancies cannot be explained only as an effect of differences in altitude above the galactic plane. 


\section{TABLE I}

\begin{tabular}{|c|c|c|}
\hline $\begin{array}{l}\text { Specific } \\
\text { distance } \\
\text { accordin } \\
\text { accessibl }\end{array}$ & $\begin{array}{l}\text { ss as a fu } \\
\text { r the Carin } \\
\text { (1970) a } \\
\text { he Milky } \\
\text { iltner (1956 }\end{array}$ & $\begin{array}{l}\text { of correc } \\
\text { aurus reg } \\
\text { the nor } \\
\text { ccording }\end{array}$ \\
\hline$m_{0}-M$ & $E \mathrm{kpc}^{-1}$ & \\
\hline & Car-Cen & NMW $^{\mathbf{a}}$ \\
\hline 7.0 & 0.338 & 1.252 \\
\hline 8.0 & 0.302 & 1.150 \\
\hline 9.0 & 0.265 & 0.900 \\
\hline 10.0 & 0.228 & 0.715 \\
\hline 11.0 & 0.192 & 0.535 \\
\hline 12.0 & 0.155 & 0.360 \\
\hline 13.0 & 0.118 & 0.180 \\
\hline
\end{tabular}

The absolute level for the NMW values is affected by the fact that most of the stars in question are particularly selected with respect to high colour excess.

${ }^{\text {a }}$ NMW $=$ Northern Milky Way.

Among other indications of the presence of small and dense units in the interstellar space we may mention the measured sizes of certain molecular radiation sources. If these formations may have dimensions of the order of some solar system radii, the dark interstellar cloud elements may also have it.

\section{Tentative Study of Density Gradients in the Interstellar Space by Means of Comparison of Colour Excess for Stars with Small Angular Separation}

\subsection{COINCIDENCES}

By visual inspection of objective-prism plates of Mily Way regions one frequently detects pairs or higher multiples of stars with very small angular separation and so closely coinciding spectral type and apparent magnitude that they form a conspicuous configuration on the plate. It might be reasonable to classify these objects, or at least a considerable fraction of them, as parts of highly diluted clusters or associations which at present can only be revealed as such coincidences - in some cases even as binaries (Stock and Wroblewski, 1972). A statistical discussion of the problem with respect to the present experience and material supports this idea. In order to establish the real physical connection between the components in each individual case it is necessary to secure additional information in the form of dynamical data etc., however.

Independently of the true genetic correlation between the 'coincidence' components, the objects in question are very useful for a large series of various investigations just because of the similarity in magnitude and spectral type in conjunction with the narrow separation. One investigation of this kind concerns the distribution of interstellar matter. The members may be easily compared with respect to parameters which are 
conditioned by the influence of this matter, for instance colour excess, polarization, or interstellar absorption lines, and local variations will be detected even in a superficial analysis. The present study is based upon UBV photometry of 320 'coincidence' groups with a total of 800 stars in the Carina-Centaurus region (Lodén, 1970). The majority of these stars are of spectral types rather close to A0 and hence, their colour excess can be estimated with fair accuracy.

\subsection{THE COLOUR EXCESS DIFFERENCES}

As a first rough approach to the problem I have computed and studied the mean values of the excess difference for certain intervals in the separation (Table IIA and IIB). If there should be a tendency towards a continuous increase of the difference with the separation over large regions, this may indicate the presence of relatively extended homogeneous clouds or moderate density gradients in interstellar space. If, on the other hand, there is no such tendency detectable one might draw the conclusion that the matter is discontinuously distributed with great density gradients within small regions. According to the table there is practically no correlation at all between mutual distance and colour excess. In other words: the chance of finding a certain difference in colour excess is the same for a very narrow pair as for an appreciably more widely separated one. Consequently, there is already some indication of the suspected distribution of the interstellar matter and the concentration in small dense units. The order of magnitude may be estimated as $10^{4} \mathrm{AU}$ or less.

A possible objection that the estimated difference might also be interpreted as a

TABLE IIA

Mean difference in colour excess as a function of lateral separation in angular distance

\begin{tabular}{rllr}
\hline Minutes of arc & $\Delta(\overline{B-V})$ & $\Delta(\overline{U-B})$ & \multicolumn{1}{c}{$N$} \\
\hline $0.05-1.0$ & 0.076 & 0.098 & 162 \\
$1.1-2.0$ & 0.070 & 0.100 & 73 \\
$2.1-3.0$ & 0.076 & 0.099 & 58 \\
$3.1-10.0$ & 0.068 & 0.100 & 158 \\
$10.1-26.0$ & 0.086 & 0.176 & 33 \\
\hline
\end{tabular}

TABLE IIB

Mean difference in colour excess as a function of lateral separation in linear distance

\begin{tabular}{lllr}
\hline $\log$ AU & $\Delta(\overline{B-V})$ & $\Delta(\overline{U-B})$ & $N$ \\
\hline$<4.0$ & 0.064 & 0.086 & 37 \\
$4.0-4.4$ & 0.084 & 0.116 & 56 \\
$4.5-4.9$ & 0.069 & 0.098 & 96 \\
$5.0-5.4$ & 0.074 & 0.096 & 158 \\
$5.5-5.9$ & 0.063 & 0.122 & 114 \\
$>5.9$ & 0.122 & 0.093 & 18 \\
\hline
\end{tabular}


TABLE III

\begin{tabular}{|c|c|c|}
\hline$m_{0}-M$ & $\Delta(\overline{B-V})$ & $N$ \\
\hline $6.0-6.9$ & 0.041 & 10 \\
\hline $7.0-7.9$ & 0.053 & 21 \\
\hline $8.0-8.9$ & 0.060 & 47 \\
\hline $9.0-9.9$ & 0.072 & 131 \\
\hline $10.0-10.9$ & 0.074 & 154 \\
\hline $11.0-11.9$ & 0.094 & 63 \\
\hline $12.0-12.9$ & 0.068 & 54 \\
\hline
\end{tabular}

difference in intrinsic colour caused by uncertainties in the classification should not be ignored. However, an effect of this type should not be dependent upon the lateral separation. It would merely increase the scatter in the individual values.

Concerning the statistical distribution of the differences in colour excess it is interesting to note that it apparently follows that of a normal distribution with maximum frequency at small values but not at zero. The corresponding distribution of the 'relative' excess differences $(\Delta(\mathrm{B}-\mathrm{V})$ /separation in parsecs) also gives the impression of a normal frequency distribution that is very similar to the just mentioned one. An investigation of the possible correlation between difference in colour excess and distance from the observer shows a rather clear statistical increase of the difference with the distance, at least up to a certain limit (Table III). This might indicate that the greater the distance, the greater the risk for obscuration of one of the two components, i.e. the result in question also speaks in favour of the discontinuous distribution of the interstellar matter.

\subsection{THE ABSOLUTE COLOUR EXCESS}

Next we may study the average behaviour of the absolute colour excess itself, represented by the mean value for both components in the same pair of stars. Starting with the frequency we then again find a rather typical normal distribution which does not change very much if we repeat the procedure with the 'specific' colour excess. Thus, there is no visible connection between the distribution of colour excesses and the distribution of stars, which one should have expected if the interstellar matter had been smoothed out along the line of sight.

Another way to study the present material is illustrated in Table IV. The investigated part of the Milky Way is subdivided in three sections, each one covering approximately $15^{\circ}$ in galactic longitude, and the average value of the excess difference is computed for each section. For comparison the corresponding average values of the total colour excess are also included. It seems evident from Table IV that there is a very poor correlation between the various parameters. The only striking feature is the apparent minimum of the excess difference in both colours for the central region. This tendency, however, has no visible correspondence in total excess or average distance. 
TABLE IV

Average values of colour excess difference for three main sections of the CarinaCentaurus region. Average total colour excess and distance added for comparison

\begin{tabular}{llll}
\hline & \multicolumn{2}{l}{ Galactic longitude intervals } & \\
\cline { 2 - 4 } & $281^{\circ}-294^{\circ}$ & $294^{\circ}-307^{\circ}$ & $307^{\circ}-320^{\circ}$ \\
\hline$\overline{\Delta(B-V)}$ & 0.078 & 0.064 & 0.078 \\
$\bar{A}(U-B)$ & 0.092 & 0.088 & 0.124 \\
$N$ & 114 & 153 & 213 \\
$\overline{E(B-V)}$ & 0.218 & 0.271 & 0.245 \\
$\overline{E(U-B)}$ & 0.128 & 0.144 & 0.136 \\
$\mathrm{Kpc}^{\mathrm{a}}$ & 1.76 & 2.10 & 1.56 \\
$\mathrm{Kpc}^{\mathrm{b}}$ & 1.30 & 1.45 & 1.14 \\
\hline
\end{tabular}

ancorrected for interstellar extinction.

b With tentative correction for interstellar extinction.

Thus there is no indication that large differences in colour excess should be connected with high values of the excess itself and vice versa. Rather there is an indication of anti-correlation, whether spurious or not. This fact might be interpreted as an effect of difference in the cloud element dimensions between different regions so that, for instance, the elements in the Crux Coal Sack region should be somewhat greater than the ones just outside this region.

Although the above observations may be interpreted in more than one manner, they all speak in favour of a very heterogeneous distribution of the interstellar dark matter unless we prefer the application of much more complicated physical procedures in the production of colour excess than the hitherto accepted ones.

In the author's opinion a continued detailed study of narrow stellar pairs with coinciding spectral type will probably be very valuable for our knowledge about the distribution of the interstellar matter.

As a preliminary result of the above discussion we may conclude that the presence of dense distinct interstellar clouds with dimensions of the order of much less than $10000 \mathrm{AU}$ should not be regarded as unreasonable. I do not even find it futile to look for observable time-variation in colour excess for some stars as a result of a combination of proper motion and heterogeneity in the interstellar obscuring medium. A corresponding study of the interstellar polarization might also be conceivable.

\section{Appendix: Hints on a Possible Model for the Distribution of Interstellar Matter}

The problem is to find within the framework of hitherto accepted values of physical parameters for the interstellar space, a mechanism that is capable of condensing matter in small units. If this turns out to give a negative result, the next problem will be to reconsider the parameters themselves without getting into conflict with generally 
accepted physical laws. As a final step, the validity of the laws themselves has to be seriously scrutinized.

For the relative vicinity of the galactic plane some tentative figures of reasonable qualitative characteristics of the average physical conditions in the interstellar space have been given and generally accepted. Although these figures are for several reasons subject to great uncertainty, it would at least be encouraging if a modified theory of any kind does not get in too much conflict with them. They do not imply, however, any absurdity in a series of substantial local deviations - even by several orders of magnitude.

In order to obtain a rough picture of one possible organization of a cloud system that is in first approximation non-hierarchical, we regard a huge homogeneous interstellar cloud the diameter of which has the order of magnitude of one kiloparsec. The cloud in question is situated in front of the observer and covers the field of vision in a certain solid angle. Its density is considered to be equal to the average density of the interstellar matter in the Milky Way. For mathematical simplicity, the cloud is regarded as spherical although its real shape is in fact completely arbitrary. Suppose the cloud is divided up in a certain number of equal fragments with the same total surface as the first one and randomly scattered within the same volume. In that case the density of the small clouds will be higher than that of the first one. Table V shows the simple relations between number, radius, and density for this situation.

For stars situated within or beyond the cloud region there is now a certain risk for obscuration by one or occasionally more clouds, but there is also a corresponding possibility that the line of sight should be free from any obstacle. Under highly idealized conditions, the fraction $F$ of the field of vision that will be obscured by $k$ clouds $(k=0-n)$ may be expressed by the relation

$$
F(k, n)=\frac{n !}{k !(n-k) !}\left(\frac{1}{n}\right)^{k}\left(1-\frac{1}{n}\right)^{n-k},
$$

where $n$ is the total number of clouds. For large $n$ values this relation will converge towards $1 / e k$ !. We may thus expect that about $1 \%$ of the number of stars beyond the

TABLE V

Correlation between number, radius, and density for constant obscuring surface

\begin{tabular}{rlll}
\hline $\log \mathrm{n}$ & $\log \mathrm{r}(\mathrm{cm})$ & $\log \varrho\left(\mathrm{g} \mathrm{cm}^{-3}\right)$ & note \\
\hline 0 & 21.5 & -26 & kpars \\
2 & 20.5 & -25 & \\
4 & 19.5 & -24 & pars \\
6 & 18.5 & -23 & \\
8 & 17.5 & -22 & conceivable region \\
10 & 16.5 & -21 & \\
12 & 15.5 & -20 & o.m. AU \\
14 & 14.5 & -19 & \\
16 & 13.5 & -18 & \\
\hline
\end{tabular}


cloud system will be obscured by 4 elementary clouds, about $6 \%$ by three, about $20 \%$ by two, about $37 \%$ by one, and about $37 \%$ by none. At a superficial comparison with the observational result these figures are apparently quite reasonable, but this statistical procedure is not realistic because the final result is not sensitive to the number of clouds. Furthermore, we have no empirical indication that the clouds should be equal in size and regular in shape, or that their total surface should be kept constant.

\section{References}

Hiltner, W. A.: 1956, Astrophys. J. 24, II.

Lodén, L. O.: 1970, Stockholm Observ. S. Milky Way Survey, Report 1.

Stock, J. and Wroblewski, H.: 1972, Astron. Astrophys. 18, 341. 